\title{
Integrated opto-chemical sensors
}

\author{
Paul V. Lambeck \\ MESA Research Institute, University of Twente, P.O. Box 217, 7500 AE Enschede (Netherlands)
}

\begin{abstract}
Integrated opto-chemical sensors have promising prospects, for example by having the potential to be realized as very sensitive small monolithic smart multisensor systems with a digital signal output. Here the main accent will be laid on the optical principles underlying chemo-optical waveguiding sensors, focusing on linear evanescent field sensors. Sensing principles and systems based on interferometry, surface plasmon resonance and luminescence quenching will be treated in more detail. Materials and technologies applied to integrated optic sensors are mentioned.
\end{abstract}

Keywords: integrated optic, chemical sensor, evanescent field, surface plasmon resonance.

\section{Introduction}

At present it is very difficult to predict, or even to indicate, which position in the chemical sensor market will be feasible for integrated optic (IO) sensors. The reason for this can be found in the vigorous state of development of the determining factors, such as the needs of the market and the performance and price of both IO chemical sensors and their main competitors, the fibre-optic, the electrochemical and the (micro-)mechanical chemical sensors.

The main markets for chemical sensors that could be considered are the processing industry, automotive industry, medicine and environmental studies. A prognosis of the qualitative and quantitative needs of the market is generally based mainly on past extrapolations; however, discontinuities are not excluded, and may arise from unpredictable processes of a political, social and even ethical nature.

Nowadays the field of chemical scnsors still contains many unknown aspects; much research is being done to improve known sensors and to invent and realize new ones. This requires a multidisciplinary approach (Fig. 1). Physics (phenomena and principles), electrical engineering (signal processing), material sciences (materials support the applied phenomena), microtechnology (producing materials in the required geometry) and

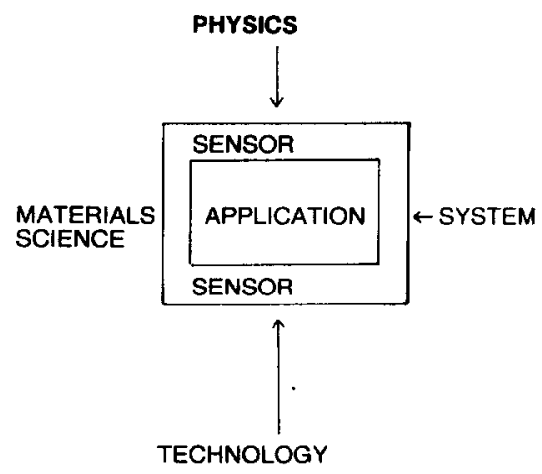

Fig. 1. Multidisciplinary approach of sensor development.

system engineering (the device itself is a system, but it is also part of a more complicated system) are involved in close mutual interaction. Also, for economic reasons, an insight into the needs of the sensor market with its numerous application areas is required.

The sensor market is too small to support intensive basic research in all of these areas. Often microsensor development in industry relies on progress made for more powerful markets, e.g., electronic ICs and, with respect to optical sensors, the telecommunications field. In creating new basic knowledge, specifically aimed at sensor development, universities can play a special role, because their research is not aimed solely at extracting maximum economic profit. 
New sensor developments may start from new findings in each of the disciplines involved, for example, from a new or improved physical principle, material or technology. The sensor field may incorporate these findings if they are relevant and will adapt them for their own particular applications if economically justified.

Many types of chemical sensors are in the first stage of development. Hence a comparison of performance and price would depend on the time and it would be premature to make general conclusions at present about their competitive power in the future. In order to obtain an insight into the relevance of a particular class of sensors, it is justified to indicate their strong and weak points in a more qualitative way. These points will be presented here for IO chemical sensors, some of them being indicated or argued in the following Sections. In order to do this, we first have to have an impression of the nature of integrated optic devices. These devices are small monolithic planar multilayer systems, acting as a complex of optical functions (optical chip). This multilayer structure can be realized by applying physical and chemical deposition and/or diffusion technologies for building the layer structure. Photolithography and etching methods are used for lateral structuring, in this way producing the waveguide channels in layouts that represent the required optical functions.

The strong points of 10 chemical sensors arise from optical sensing, and the use of waveguides and integrated structures. By sensing optically, electromagnetic interference and the danger of explosion are (almost) excluded. As well as this, optical methods often offer a high sensitivity. By using waveguides the light path can be controlled simply, without the necessity of using bulky components like mirrors or beam splitters. Also propagation of light through waveguides offers excellent possibilities of influencing its optical characteristics both for sensing and processing purposes. This latter facility is in fact the origin of the interest of the (optical) telecommunications field in integrated optics, an interest that will be very beneficial in the field of optical sensors.

The use of integrated optic systems instead of fibre-optic ones adds another set of strong points. There is a large flexibility in the choice of construction materials and dimensions. The systems are small and rigid and by using silicon wafers as substrates, integration of optical and electrical functions can be achieved, offering the prospect of monolithic smart multisensor systems. It also has economical advantages, which are common for most microtechnological devices: a batch-wise mass production, in which all basic functions required for the sensing system can be realized simultaneously.

However, the rather complicated smart multisensor systems are still in an initial state and a lot of research will have to be done before they will be ready for production. On that long way, useful spin-off in the form of less complicated IO sensors is expected and in some cases already realized. The IO chemical sensor, in general, will not be very cheap, although of course its price will decrease with the increasing number of sensors produced. They are expected to be more expensive than chemical ISFET sensors, hence, their use may be restricted to applications where ISFET sensors cannot meet the specifications.

This paper has been structured as follows: for readers not familiar with optical waveguides, a short description of the waveguide phenomenon is given in Section 2, already focusing on aspects relevant for applications in chemical sensors. In Section 3 chemo-optical waveguide sensors are subdivided into classes according to the sensing mechanism. The chemo-optical transduction layer effective in interrelating chemical and optical domains is introduced in Section 4. In Section 5 read-out principles and circuits are treated, paying special attention to (Mach-Zehnder) interferometry $[1,2]$, surface plasmon resonance $[3-5]$ and luminescence quenching $[4,6]$. The influence of disturbing factors is discussed in Section 6. Materials used in IO sensors are mentioned in Section 7. Finally in Section 8 the main conclusions are summarized.

\section{Waveguides and waveguiding [7]}

A well-known waveguide is the glass fibre, consisting of a core with a diameter as small as $5 \mu \mathrm{m}$, which is surrounded by a cladding having a lower refractive index. Thus light rays that are incident on the boundary of the core and the 


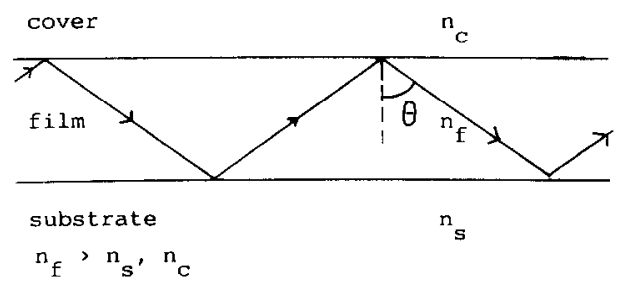

Fig. 2. Glass fibre, ray picture of waveguide.

cladding from the core side at an angle of incidence larger than the critical angle will be totally reflected (Fig. 2). The corresponding light will be approximately confined to the core and will propagate effectively along the direction of the fibre.

In integrated optics the waveguide has another shape. The slab waveguide, for example, consists of a thin core layer (thickness in the range 0.1 $5 \mu \mathrm{m}$ ) applied on or in a substrate by microtechnologies such as deposition, sputtering, chemical vapour evaporation, diffusion, etc. This core layer will be sandwiched between layers having a lower refractive index (the upper layer may be air). Thus light can be confined in the direction perpendicular to the layers.

A lightwave channel is made by confining the light in the lateral direction too. For this purpose the channel region has to have an (effectively) higher refractive index than all its surroundings. This can be realized in various ways, for example, by etching away a thin layer from the region outside the channel, leaving behind a strip-shaped waveguide, called a ridge guide (Fig. 3 ). Generally the width of these channels is in the range $2-10 \mu \mathrm{m}$. Just as in the case of the fibre, the guided light can follow the channel, even if it has been bent.

For a more quantitative description we leave the simple ray picture sketched above and apply

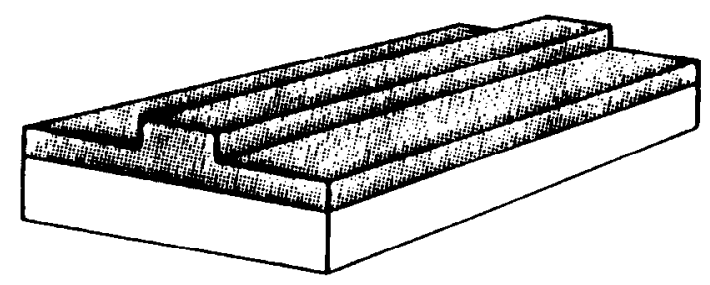

Fig. 3. A waveguide channel shaped as a ridge guide.

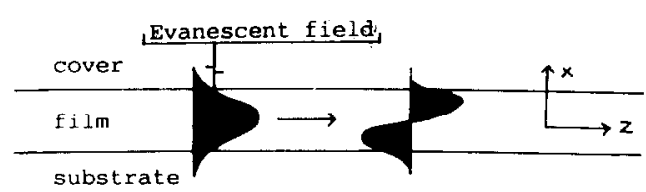

Fig. 4. Field profiles of slab guide modes 0 and 1 .

Maxwell theory. Starting from Maxwell's equations and introducing the proper boundary conditions, it has been deduced [7] that for each wavelength light can only propagate through the guide according to particular patterns of the electromagnetic field, called guided modes.

In a slab waveguide (Fig. 4) the electrical component of the field is given by the relation

$\bar{E}_{i}(x, z, \omega, t)=\bar{E}_{i}^{0}(x, \omega) \exp \left[\mathrm{j}\left(\omega t-\widetilde{\beta}_{i} z\right)\right]$

The field profile $\bar{E}_{i}^{0}$ and propagation constant $\widetilde{\beta}_{i}$ of mode $i$ are dependent on the angular frequency $\omega$, the geometry and the dielectric constant $\varepsilon$ of all the materials of the slab guide. By proper control of these parameters, monomode waveguiding systems can be realized having only one TE and TM guided-wave solution of Maxwell's equations for each wavelength.

The field does not appear to be strictly confined to the core layer, but also shows exponentially decaying tails in the surrounding materials, i.e., the evanescent fields. The decay length ranges from one tenth of the applied wavelength to infinity, depending on the geometry and applied materials. This evanescent nature of the field outside the core is characteristic of all guided modes. In a channel waveguide the field profile, now being a function of both lateral coordinates, decays outside the channel exponentially in all directions. One or both sandwiching layers can be replaced by a stratified multilayer structure with all component materials showing refractive indices lower than the core index. The evanescent nature of the field is essentially maintained; the field decay in each layer, however, is now given by its particular value of the decay constant. As well as guided modes, Maxwell's equations also offer radiation modes. These are plane waves crossing the core layer with angles of incidence smaller than the critical angle of one of the layer couples. 


\section{Sensing principles in waveguiding system; a classification}

\subsection{Extrinsic or intrinsic}

In some waveguiding sensors waveguides are only used for easy transport of light to and/or from a sensing region (which is located outside the waveguiding structures (Fig. 5)). This type of optical sensor is called extrinsic. Here light radiating out of fibre 1 or generated in the sensing region is partially trapped by fibre 2 as guided modes. The transfer function can be modulated by the sensing action, e.g., absorption by a species with unknown concentration. Here it is interesting to note that this extrinsic sensor can be used for more spectacular purposes too, for example, for providing an optical read-out of the displacement of a membrane in a micromechanical pressure sensor [8]. The counterpart of extrinsic sensors is formed by the intrinsic ones, in which sensing is achieved in regions within the region of control of the guided modes.

\subsection{Generator or modulator}

Light is needed for the optical sensing of chemical concentrations. This light is supplied by the sensing mechanism itself in sensors of the generator type. This light generation relies on chemical reactions in which the molecules of the measurand (i.e., the species $X$ whose concentration has to be detected) are involved. One of the reaction products is created in an excited state and is capable of emitting light during return to the ground state. The intensity reflects the concentration $C_{\mathrm{X}}$. The measured molecule $\mathrm{X}$ may act as a reactant [9]:

$\mathrm{A}+\mathrm{X} \rightarrow \mathrm{L}+\mathrm{M}^{*}$

or as a catalyst:

$\mathrm{A}+\mathrm{B} \stackrel{\mathrm{x}}{\rightarrow} \mathrm{L}+\mathrm{M}^{*}$

where
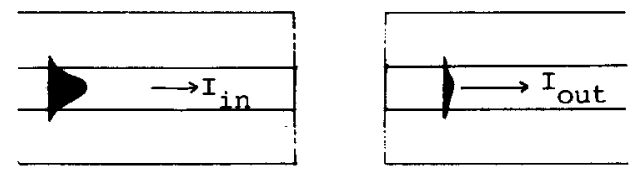

Fig. 5. A configuration for an extrinsic sensor.

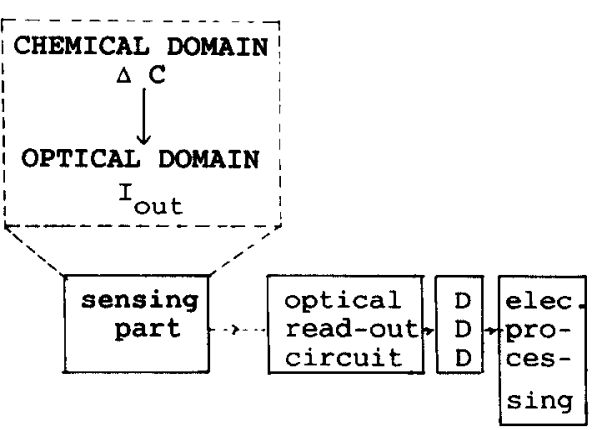

Fig. 6. Block scheme of a generator-type sensor system.

$\mathbf{M}^{*} \rightarrow \mathbf{M}+h v$

In the first case the species $X$ is gradually consumed in the latter until its concentration remains constant. Of course all (other) reactants have to be added deliberately. Here the optical system only has the task of collecting the emitted light and transporting it to a detector. An intrinsic sensor shows a much larger collection efficiency than an extrinsic one due to special properties of the mode fields.

Generator-type sensors are characterized by the transduction chain

$\Delta C \rightarrow \Delta I \rightarrow \Delta I^{\prime} \rightarrow \Delta V$

which can be effected by the system shown symbolically in Fig. 6.

In modulator-type chemo-optical sensors, the species $\mathrm{X}$ induces a change of an optical parameter, which modulates a guided wave that propagates through the sensing part.

\subsection{Core or evanescent field}

The optical phenomena that provide sensing may occur in the core layer or outside it. Essentially the former option is the more sensitive one, because that area is probed by a major part of the mode energy. Hence, for example, in an electric field sensor it is the core layer that shows the electro-optic cross effect on which the sensor relies. Some IO chemical sensors have been reported in which core properties can be modulated [10]. A condition is that there is some porosity of the core material, permitting its invasion by the chemical species. Up to now this prerequisite has excluded fibre-optic sensors from this sensing class. Specifications as to reversibility 
nd response time will often disfavour this type of insing.

In most chemo-optical sensors the sensing acon is localized in the cladding region and is robed by the evanescent field: evanescent field znsors. In IO sensors the evanescent field region is pen to the medium containing the measurand. In rder to use fibres, part of the cladding has to be imoved locally by polishing $[11,12]$ or a special rpe of fibre, the D-shaped fibre [13], has to be pplied.

\section{.4. Linear or non-linear}

Sensing by modulation can be based on linear $r$ non-linear phenomena. If during sensing guided lodes with another angular frequency $\omega_{j}$, differ$\mathrm{nt}$ from that of the incoming mode $\omega_{i}$, are generted (Raman effect, luminescence), then the sensor an be called non-linear. In linear sensors the :equency remains constant, but the polarization tate, amplitude or phase of the guided mode is hanged as a consequence of the concentration1duced change of the dielectric constant of one of ae compositional materials.

In these linear sensors the sensing part is folswed by an optical read-out part, which has the ask of transforming the change in mode parameir into an appropriate intensity change. Often the ensing part is preceded by an optical-conditioning art that extracts from the light offered by the ght source the component(s) which is (are) atched to the principles applied in sensing and ead-out. This sensing system is presented scheratically in Fig. 7. The corresponding transducion chain is

$$
\underbrace{C \rightarrow \Delta\left(\text { Par }_{\text {opt }}\right) \rightarrow \Delta\left(P a r_{\text {mode }}\right) \rightarrow \Delta I}_{\text {Sensing part }} \underbrace{\Delta I \Delta V}_{\substack{\text { read-out } \\ \text { part }}}
$$

here Par $_{\text {opt }}=$ an (up to now undefined) optical arameter in the sensing part and $P a r_{\text {mode }}=\mathbf{a}$ haracteristic mode parameter.

In non-linear modulating sensors the evanescent eld has a double function. The active particles are dmitted to the evanescent field region (Fig. 8) nd are excited by the evanescent field of the acoming mode. Also in collecting the emitted ght the evanescent field plays a special role, corcing the excited particles to emit a remarkable art of their radiation in guided moles [14]. The

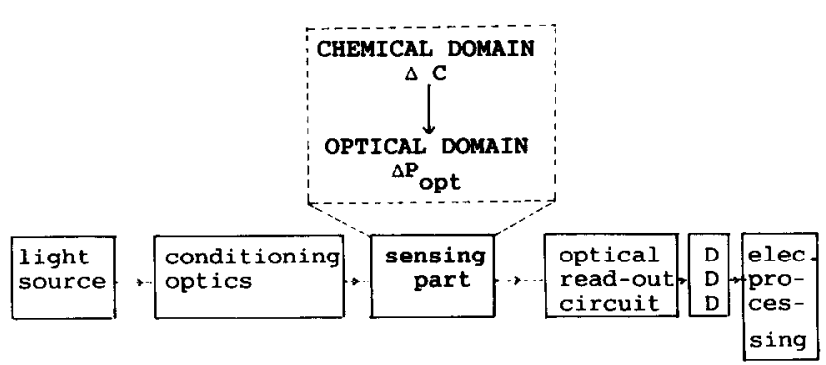

Fig. 7. Block scheme of a modulator-type sensor system.

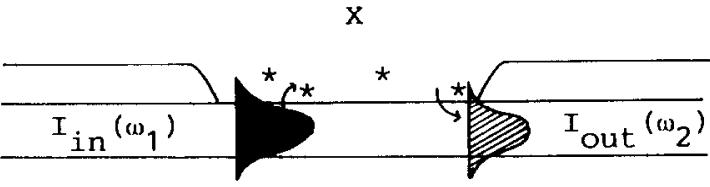

Fig. 8. Sensing part of a non-linear sensor.

absolute yield can be increased by enlarging the interaction length $L$. These sensors require functions to separate emitted and incoming light. The effectiveness of this separation strongly codefines the ultimate limits of sensitivity. Although these non-linear modulating sensors are very attractive because of the large sensitivity that can be obtained, they will not be treated in more detail.

\subsection{Refractive or absorptive}

In linear sensors the presence of a chemical species $\mathrm{X}$ effects a change of the dielectric constant of one of the layers. This dielectric constant can be presented as a complex quantity $\tilde{\varepsilon}=\varepsilon^{\prime}-\mathrm{j} \varepsilon^{\prime \prime}$; for optical frequencies $\varepsilon^{\prime \prime}$ determines the absorption losses and $\varepsilon^{\prime}$ in lossless (both $\varepsilon^{\prime}$ and $\varepsilon^{\prime \prime}$ in lossy) media is related to the refractive index $n$.

A further subdivision of linear sensors has been based on the exploitation of concentrationinduced changes of either the real part $\varepsilon^{\prime}$ or the imaginary part $\varepsilon^{\prime \prime}$ of the dielectric constant. The corresponding sensor types are called refractive and absorptive, respectively. The $\tilde{\varepsilon}$ changes will cause a change of the complex propagation constant $\widetilde{\beta}=\beta^{\prime}-\mathrm{j} \beta^{\prime \prime}$, in which $\beta^{\prime \prime}$ represents the attenuation of the mode. The task of the read-out part can now be made more concrete: transforming this $\tilde{\beta}$ change into an intensity change. Some of the read-out principles will be treated in detail in 


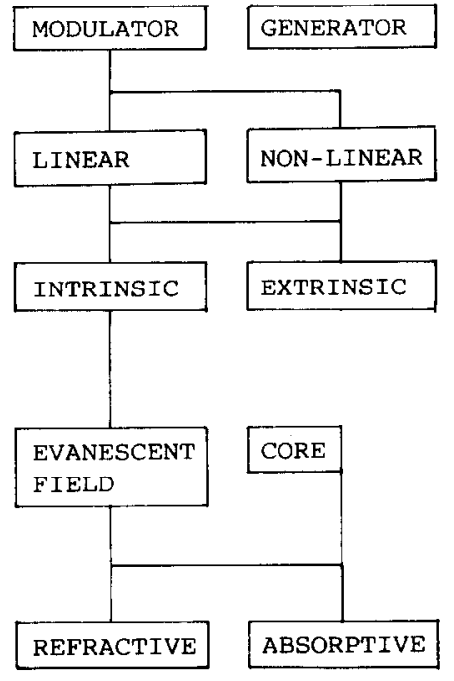

Fig. 9. Subdivisions of chemo-optical sensors.

Section 5. The various classifications are summarized in Fig. 9. In this paper we shall go further with linear evanescent field sensors only, but before doing do we have to introduce the chemooptical transduction layer, a frequently used intermediate region between the chemical and optical domains.

\section{The chemo-optical transduction layer}

In chemo-optical evanescent field sensors, the medium containing the $X$ molecules is introduced into the evanescent field region. Here the $\tilde{\varepsilon}$ change caused by the presence of these molecules is probed by the mode, resulting in a $\widetilde{\beta}$ change in the sensing region, defined by the interaction length $L$. However, a $\Delta \widetilde{\beta}-\Delta C_{\mathrm{X}}$ relation can only be applied if we are sure that unknown concentration variations of other species cannot interfere. This will be true for a gas $\mathrm{X}$ or a solution in which $\mathrm{X}$ is the only solute. If that condition is not fulfilled, selectivity has to be introduced, for example, by excluding the presence of disturbing species in (well-defined parts of) the evanescent field region.

In order to make the sensor more sensitive, it would be interesting to concentrate the $\mathrm{X}$ particles in the evanescent field region and/or to amplify their optical effects by chemically modifying them. Selectivity, concentration and amplification may all be obtained by introducing an appropriate

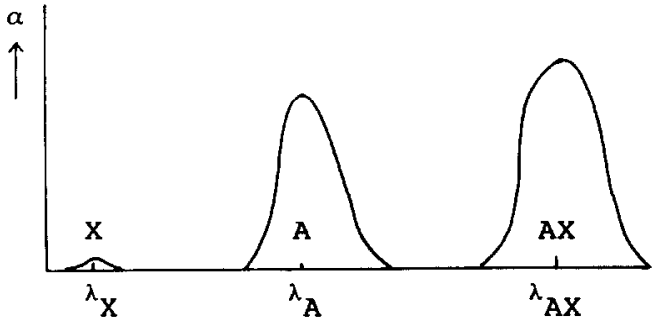

Fig. 10. Absorption bands of bare $X$ particles, $A$ and $A X$ molecules.

chemotransduction layer, applied between the core layer and the $\mathrm{X}$-containing evironment. This layer will contain immobilized molecules $A$, capable of associating selectively and preferentially reversible with $\mathrm{X}$ molecules.

$\mathrm{A}+\mathrm{X} \underset{k_{2}}{\stackrel{k_{1}}{\rightleftharpoons}} \mathrm{AX} \quad K_{\text {ass }}=k_{1} / k_{2}=[\mathrm{AX}] /[\mathrm{A}][\mathrm{X}]$

Although only the replacement of solvent molecules by $\mathrm{X}$ molecules creates optical changes, a higher gain can be obtained if the contributions of the A and AX molecules to the dielectric constant of the layer differ greatly. In fact special A molecules often have to be designed for maximizing this difference. In absorptive sensors strongly absorptive A molecules showing large solvatochromic effects are desired. In that case association will result in a large wavelength shift of an intense absorption band. It can be concluded from Fig. 10 that by using $\lambda_{\mathrm{Ax}}$ as probing wavelength instead of $\lambda_{\mathrm{x}}$, we have obtained amplification. The number of A molecules that will be associated depends on the $\mathrm{X}$ concentration in a characteristic way (Fig. $11)$. The association constant $K_{\text {ass }}$ defines the centre of the dynamic range of the sensor, a range that is restricted to merely two to four decades. The reaction rate constants $k_{1}$ and $k_{2}$ codefine response times. We note that a larger value of $K_{\text {ass }}$ is related to a larger $k_{1}$ and/or a smaller $k_{2}$. The

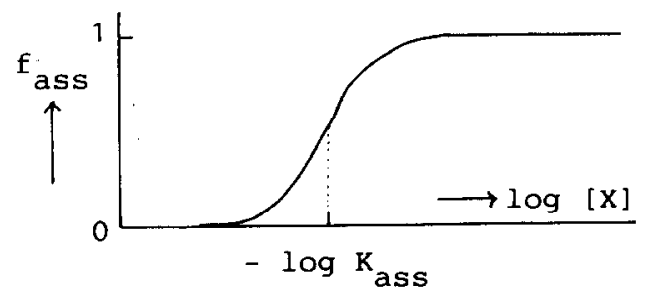

Fig. 11. Associated fraction $f_{\text {ass }} \equiv[\mathrm{AX}] /([\mathrm{A}]+[\mathrm{AX}])$ as a function of $[\mathrm{X}]$. 
TABLE 1. Classification of chemo-optical sensor properties according to their origin

\begin{tabular}{lll}
\hline Property & $\begin{array}{l}\text { Transduction } \\
\text { layer }\end{array}$ & $\begin{array}{l}\text { Optical detection } \\
\text { system }\end{array}$ \\
\hline $\begin{array}{l}\text { Selectivity } \\
\text { Dynamic range }\end{array}$ & $* *$ & \\
$\quad$ centre & $* *$ & \\
$\quad$ magnitude & $*$ & $*$ \\
Sensitivity & $*$ & $*$ \\
Resolution & $*$ & $*$ \\
Response time & $* *$ & $*$ \\
Calibration & $(* ?)$ & $*$ \\
Reproducibility & $*$ & $*$ \\
Stability & $*$ & $* *$ \\
Costs & $*$ & .
\end{tabular}

latter manifests itself as a slower dissociation rate, however, and would be at the cost of the response time. That response time also depends on the diffusion rate of $X$ molecules both in the solution and the transduction layer. A fast response generally requires a thin transduction layer, varying from monomolecular to a thickness of some tenths of a micron. This latter thickness is of the order of magnitude of the decay length of the evanescent field and gives rise to fruitful waveguide optimization. In Table 1 relevant chemo-optical sensor properties are classified according to their origin, either a transduction layer or an optical system.

Sometimes the chemo-optical transduction layer can be made from known materials. As an example, for refractive sensing we would like to mention the layers applied in chemo-optical immunosensors [3, 15]: immobilized antibodies, which selectively associate with specific antigens. Both materials are transparent in the visible region. Hence the refractive index will change locally because associated antigens have dispelled solvent molecules. An example of a transduction layer for an absorptive sensor is the Bromo Cresol Purple layer, used for ammonia detection [16].

Often, however, new molecules with a performance well matched to the measuring problem have to be designed (molecular engineering) and synthesized. In Fig. 12 we present an example of a chromoionophoric molecule showing a strong preference for association with $\mathrm{Ca}^{2+}$ ions [5, 17]. This association appears to result in a $90 \mathrm{~nm}$

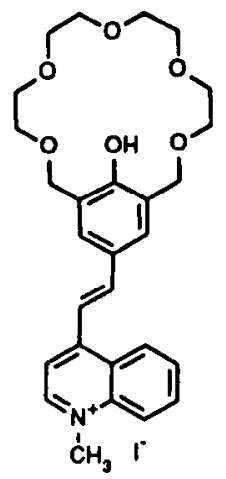

Fig. 12. Chromoionophore based on a phenolic crown ether and a merocyanine dye.

wavelength shift of a rather narrow absorption band. The molecule consists of an ionophoric part (the crown ether in which the $\mathrm{Ca}^{2+}$ ion can be trapped) and a dye as chromophoric part. The bandshift results from the change of the electronic structure of the dye caused by the capture of the ion. Introduction of particular functional groups in the crown ether may modify the association constant.

If a solution contains several known compounds the selectivity problem can be simplified. A number of interface layers, at least equal to the number of compounds, is required, each layer showing different affinities for each of the ions. By using numerical methods, the concentrations can be calculated from the signals [18].

We emphasize that association and immobilization problems are common to transduction layers for all types of chemical sensor. The only task that is specific for optical sensing is the introduction of functional optical groups in the sensing active molecules.

\section{Read-out principles}

\subsection{Introduction}

Here we focus on linear evanescent field sensors. They rely on chemically induced changes of the dielectric constant. A change of the real part of the dielectric constant gives a change in the real part of the propagation constant, showing itself as a change of the phase of the mode. This phase change can be transformed into an intensity change by use of the optical interference phenomenon (for example, in a Mach-Zehnder interferometer [2]). In 
some read-out methods the interference phenomenon manifests itself as a resonance: the FabryPerot resonator, ring resonator and all methods based on mode coupling (e.g., surface plasmon resonance [3-5] and grating-assisted coupling into radiation modes [19]).

Changes of the imaginary part, $\varepsilon^{\prime \prime}$, of the dielectric constant will directly result in changes of the attenuation of the mode, which can be measured as the ratio between input and output intensities [20]. Also more sophisticated methods have been developed, like luminescence quenching $[4,6]$ and the application of the absorption dependency of the finesse of Fabry-Perot resonators [21].

All linear evanescent field sensors are of the modulation type. Hence as well as the output signal a reference signal is required, for example, for defining the zero point. For absorptive sensors it is the input intensity that is needed for referencing; this value is usually obtained by guiding part of the incoming light into a reference channel. In principles based on mode-coupling, modifications exist that make use of the shift of an angle that is characteristic for an intensity extremum [3]. They require knowledge of a reference angle related to a known concentration.

Now we shall treat some read-out principles and corresponding read-out systems in more detail. In this Section we shall not consider disturbant factors such as noise, temperature sensitivity and technological inaccuracies. These will be discussed later on. The first and second principles, interferance according to the Mach-Zehnder scheme and surface plasmon resonance, are essentially of the refractive type and $\beta^{\prime}$ changes have to be read out. Both principles are well known from bulk optics. We shall show how the corresponding bulky equipment can be miniaturized by the use of integrated optics, resulting in smaller more rigid and cheaper measuring systems. The third principle, luminescence quenching, is an absorptive one and no bulky pendant is known.

\subsection{Mach-Zehnder interferometer}

A bulky set-up of the Mach-Zehnder interferometer is sketched in Fig. 13(a). An incoming coherent monochromatic light beam is split into two beams of equal intensity. By means of mirrors, both beams are again combined at a second beam splitter, where they interfere. For a perfect

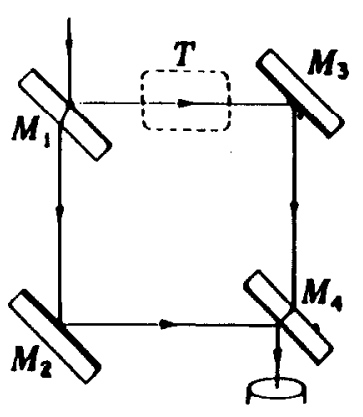

(a)

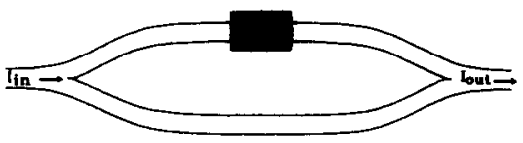

(b)

Fig. 13. A Mach-Zehnder interferometer in a bulky set-up (a) and in an integrated optic version (b).

interferometer the transfer function $I_{\text {out }} / I_{\text {in }}$ is determined only by the phase difference $\varphi$ between both beams when meeting again: $I_{\text {out }} / I_{\text {in }}=\cos ^{2} \varphi / 2$. Changes in the local dielectric constant in one of the branches will be manifested as a change of $\varphi$. In Fig. 13(b) the top view of the integrated optic version has been depicted, showing a waveguiding circuit. There is no need for mirrors, because the light path is controlled by the waveguide channels. The function of the classical beam splitters has been taken over by the Y-junctions. Optical circuitry occupies an area of about $1 \mathrm{~cm} \times 0.01 \mathrm{~cm}$; input and output beams can be transported by fibres.

By locally applying a chemo-optical transduction layer on top of one of the branches, we obtain a chemo-optical sensor. Its action can be represented by the transduction chain:

$\Delta C_{\mathrm{X}} \rightarrow \Delta \varepsilon^{\prime} \rightarrow \Delta \beta^{\prime} \rightarrow \Delta \varphi \rightarrow \Delta\left(I_{\text {out }} / I_{\text {in }}\right)$

\subsection{Surface plasmon resonance ( $S P R)$}

A serious drawback of the evanescent field sensing that has been described up to now is the low probing efficiency. Light propagating only as an evanescent field feels the $\varepsilon^{\prime}$ change of the transduction layer, its energy being merely a few percent of the total mode energy. If this percentage could be increased, $d \beta^{\prime} / d \varepsilon^{\prime}$ would grow as well. This can be accomplished by using the surface plasmon.

The surface plasmon is a guided mode, propagating along the interface of a metal such as silver 


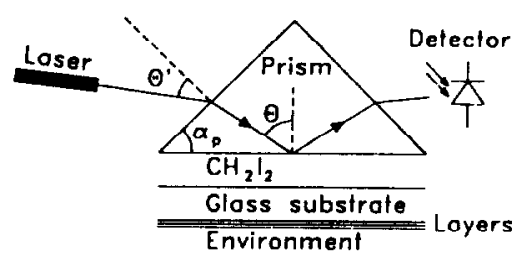

(a)

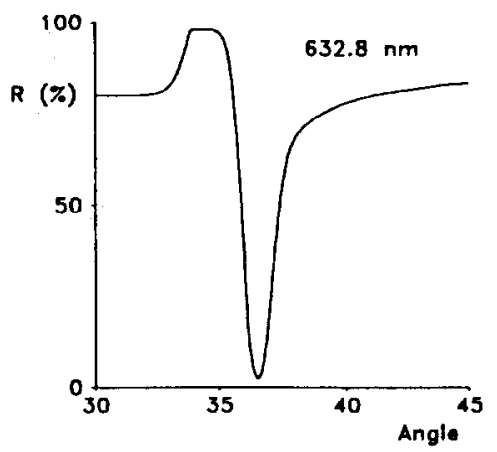

(b)

Fig. 14. (a) Kretchmann configuration. (b) Reflection curve $R(\theta)$.

and a dielectric [22]. By taking the transduction layer for the dielectric, nearly half of the mode energy will propagate through that layer, resulting in a greatly increased probing sensitivity. However, it is not possible to apply these surface plasmons in an interferometer. The plasmon mode is strongly attenuated (showing a decay length of merely $10-20 \mu \mathrm{m}$ ) because the other half of the energy propagates through the very lossy silver layer. Nevertheless, the propagation constant can be read out, but in order to do this we have to rely on mode coupling.

In the bulky Kretchmann configuration [23] (Fig. 14(a)) the coupling between the incident light beam and the surface plasmon is used. In fact the silver layer shows two surface plasmons, one on each side. By applying a very thin silver layer ( $50 \mathrm{~nm}$ thickness), coupling to the plasmon that propagates along the silver-transduction layer interface is feasible. Generally a light beam incident on the silver layer will be totally reflected. However, if the coupling condition $\beta_{\mathrm{sp}}=(2 \pi / \lambda) \sin \theta$ is fulfilled, this reflection will be frustrated because incident light will now couple to the plasmon and it will be dissipated by the silver layer. Because of the lossy character, this coupling is spread over a small $\theta$ interval. Thus a resonance dip in the $R(\theta)$ reflection curve will be observed (Fig. 14(b)). This

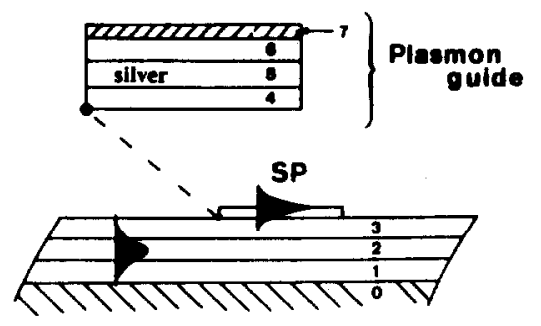

Fig. 15. Schematic cross section of an SPR waveguide sensor. 2, SiON waveguide; 5, SP-supporting metal layer; 7 , transduction layer; $1,3,4,6$, buffer layers.

dip will be shifted along the $\theta$-axis as a consequence of $\varepsilon^{\prime}$ changes in the transduction layer.

The reflection curve can be obtained experimentally by rotating the sample. Here some other methods will also be presented, mainly because they can be exactly implemented in integrated optic versions as well. By using a slightly convergent beam, the complete reflection curve can be obtained instantaneously by imaging the reflected light on a diode array [24]. The introduction of a vibrating mirror offers the possibility of transforming dip shifts into time differences which can be measured more accurately [25].

In integrated optic versions the coupling between guided modes and the surface plasmon is exploited $[4,6]$. This coupling will occur if $\beta_{\mathrm{sp}}=\beta_{\text {waveguide }}$. For this purpose we apply a surface-plasmonsupporting multilayer structure on top of a waveguide (Fig. 15). Modes of various propagation constants are launched into the waveguide, either all together or one at a time.

In a first design [4] a band of $\beta$ values is offered to the plasmon by sending polychromatic light into the monomode waveguide. Each wavelength corresponds to a particular $\beta$ value. The mode with a wavelength obeying the coupling condition will be missing at the output. In order to determine this wavelength, the light is coupled to radiation modes using a grating with a period $\Lambda$. Because now the coupling condition

$\beta_{\text {wavelength }}=\frac{2 \pi}{\Lambda}+\frac{2 \pi}{\lambda} \sin \theta$

has to be obeyed, the outcoming radiation modes are spectrally resolved and the complete reflection curves can also be obtained instantaneously by imaging on a diode array.

The need to couple light out of the waveguide can be avoided by using the configuration depicted 


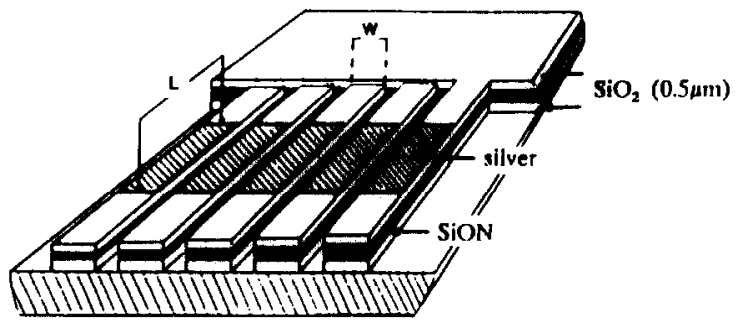

Fig. 16. Schematic drawing of an SPR-waveguide array sensor.

in Fig. 16 [4]. It consists of an array of channel waveguides with different cross sections, each provided with a transduction layer. Fed with monochromatic light, each channel will support a mode with a particular $\beta$ value. The reflection curve is now obtained as an intensity distribution over the different channels. By applying the optical circuitry on an Si wafer, the required detectors can be implemented in the wafer itself.

The most promising design is, in our opinion, a configuration in which, just as in the case of the vibrating mirror, the dip shift is transformed into time differences (Fig. 17). The system consists of a network of monomode guides on an $\mathrm{Si}$ wafer in which detectors and electronic circuits are implemented. A cheap broad-band laser diode (BBL) is mounted on the chip as a light source. TE modes, being superfluous because only TM modes are effective, are drawn off by using an asymmetric Y-junction (MSY). A dynamic wavelength filter (DWF) offers an output of which the wavelength varies with time in a well-defined way. Next the light enters a symmetrical Y-junction ( where it is divided over two channels, one su] ing the SP structure and the other for refere The concentration can be obtained by elec processing of the detector outputs. We not this configuration may also be very appro for miniaturizing the output grating couple posed by Lukosz et al. [7].

For measurements under laboratory conc such as immunosensing, bulky SPR equip provided with cheap disposable surface-pla supporting multilayer structures, may be ve propriate. The integrated optic versions, esp the one where detectors and electronic-proc circuits are also integrated, seem to be mo tractive for use as stand-alone systems.

\subsection{Luminescence quenching}

The sensing part of the most simple abso sensor is given in Fig. 18. It is a simple $s$ with a good performance if optimized. Nev less, we introduce another type of absorptiv sor, namely one based on luminscence quer (Fig. 19) $[4,6]$, which may show a better $F$ mance. The key to this sensor is exciton ho] i.e., a radiationless energy transfer betwee centres. The luminescent material has been $b$ such a way that energy before being emitte migrate along a long distance, stochastically ping from one (luminescent) centre to a bouring one. The transduction layer consi molecules showing in an associated state a sorption band that is matched to the excitc

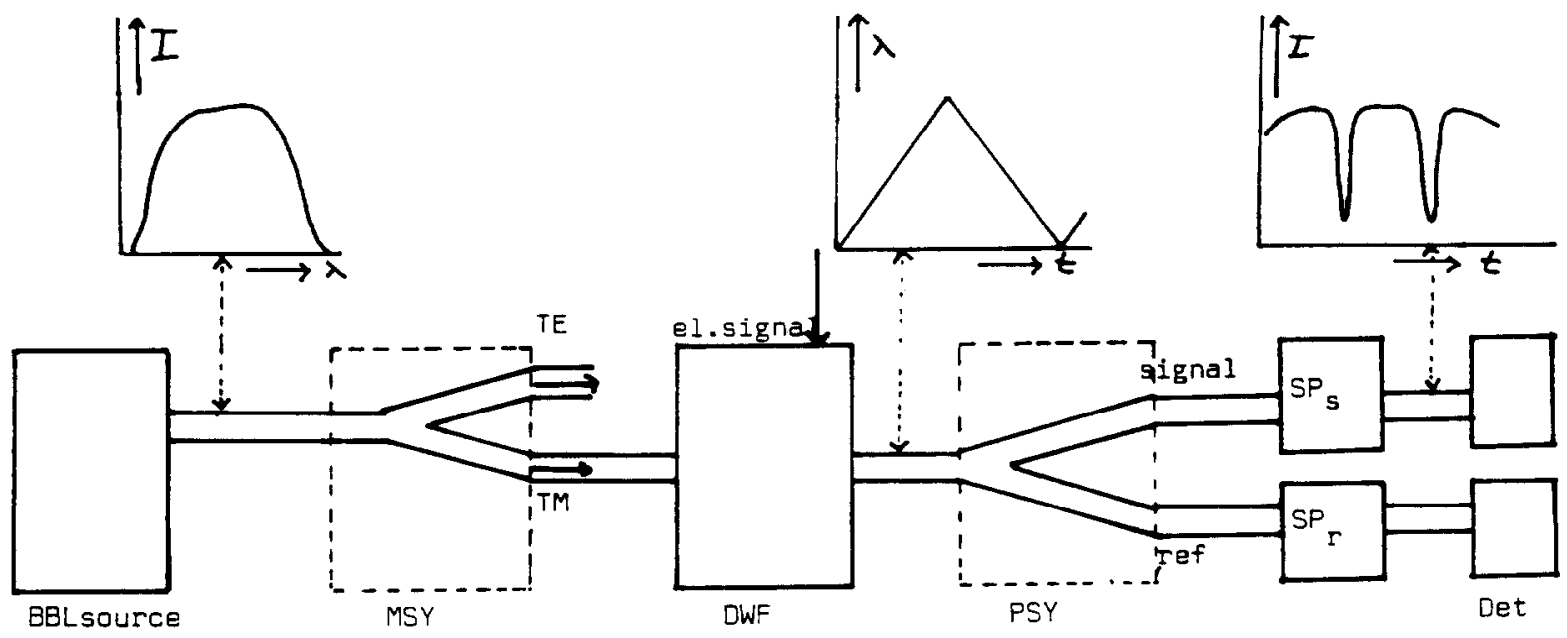

Fig. 17. Potential IO sensing system, based on surface plasmon resonance. 


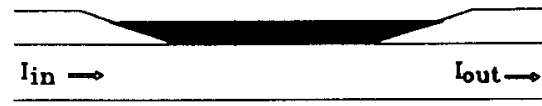

Fig. 18. Simple absorptive sensing part.

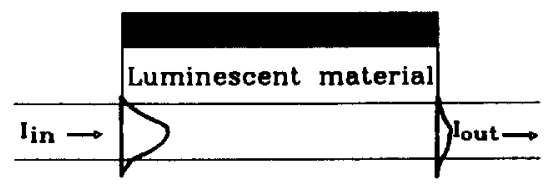

Fig. 19. Sensing part of a sensor based on luminescence quenching.

ergy. Thus they are able to trap a neighbouring exciton; if they are also able to return to the ground state radiationless, the exciton energy will now be dissipated. If the time required for this return is small compared to the relaxation time of light emission by the luminescent molecules, one associated molecule can trap a large number of excitons. Because of the antenna action of the trapping-associated molecule, the sensing is equivalent to probing a much thicker layer, and amplification has been obtained. Because of the short range of this hopping, generally only a layer $50-100 \AA$ thick will be probed. Thus there is no interference by other types of molecules that might be present in the neighbouring solution.

\section{Disturbances}

The various chemo-optical principles as presented in preceding Sections may be compared with respect to the signal (intensity change) arising from a given concentration of $C_{\mathbf{X}}$. However, for sensitivity and resolution not the signal itself but the signal-to-noise ratio is relevant. Hence sources of noise have to be traced. In general, disturbances may arise from physical flutuations as well as from the imperfectness of the technology, causing a spread in dimensions and physical constants. Some of the disturbance factors can be compensated by proper referencing. This concerns temperature fluctuations and the unwanted variations of the dielectric constant of the environment caused by other compounds, which generally will also be probed.

In principle, the use of reference channels showing the same temperature fluctuations and pro- vided with a well-matched dummy layer will be very fruitful. Here the Mach-Zehnder interferometer is very interesting, because the second branch, at a distance of about $50 \mu \mathrm{m}$ from the signal branch, offers itself as a natural candidate for this compensation function. However, if the temperature dependency of $K_{\text {ass }}$ were important, insertion of a temperature sensor would be necessary.

Sacrificing the response time, contamination can be prevented by screening the transduction layer from its environment by a (vibrating) membrane permeable by the measurand. Contamination influences can be reduced by referencing using a second guided mode [26]. Intensity variations of the light source can be taken into account by leading a part of the incoming light into an extra reference channel.

Detector noise depends more or less on the price. Expensive detection equipment capable of photon counting may even utilize all the inherent sensitivity of generator-type sensors.

Technological tolerances make it impossible to realize exactly the goal that has been aimed at, for example, a waveguide channel showing precisely the prescripted value of the propagation constant. The relevancy of these technological uncertainties depends on the type of basic function. On the other hand, their magnitude is strongly dependent on the material and technology applied (see Section 7). Thus the choices of material and technology are closely related to the basic functions for which the optical circuitry has to be built and the application of exciting basis functions has to be avoided as much as possible. The exact thickness of the waveguiding layer is not relevant for a Mach-Zehnder interferometer, although thickness uniformity of the Y-junction is desirable. For functions such as a grating coupler, however, this value is essential.

If necessary, optical functions can be tuned individually, e.g., laser trimming of a $3 \mathrm{~dB}$ coupler realized by $\mathrm{SiON}$ technology [27, 28]. Also crosseffects such as the electro-optic effect and the thermo-optic effect may be applied for tuning, although the tuning range is small. Using electrooptic materials, refractive indices can be changed by about $0.1 \%$ and by using thermo-optic effects about a $1 \%$ change is feasible. Another way to reduce the effects of technological tolerances is by looking for sets of parameter values that 
make the function less sensitive for their inherent uncertainties. For this purpose numerical calculations for simulating the performance are indispensable.

\section{Materials and technologies}

It cannot be expected that for a given compound the properties of a thin film are identical to those of the crystal. Compounds which are very transparent as a single crystal may give very lossy waveguides if made as a thin film. Absorbing impurities may be built-in as a consequence of the applied microtechnology. A columnar microstructure may give large surface roughness and voids in the film, which cause disastrous scattering losses. The quality of the channels also depends strongly on the applied (etching) technology.

Because of position-dependent and fluctuating processing conditions, inhomogeneities may arise, leading to a non-uniform thickness and dielectric constant. Stresses may develop during cooling to room temperature as a consequence of differences in thermal expansion coefficients. This will give rise to inhomogeneities and (extra) anisotropy. Hence waveguide properties always show an undesigned spread in values, both in one circuit (homogeneity) and from batch to batch (reproducibility). The values themselves and their spread are not a characteristic of the compound but of a film made from that compound by a given technology.

The sensor market has been pampered in the past; generally sensors are cheap and chemo-optical sensors are also required to be cheap. This may exclude the use of $\mathrm{LiNbO}_{3}$ and III-V compounds, both fruitfully applied in optical devices for telecommunications. With regard to the price, circuits made of indiffused glass or dipped or spun polymers are more attractive because both the compounds and the applied technologies are relatively cheap.

At present there is a good understanding and control of the processes going on during the production of indiffused glass waveguides and they can be achieved with an uncertainty of the propagation constant of the order of magnitude of $10^{-2} \%$ [28]. Here sodium ions in the glass are locally (defined by a metal mask applied on the glass) replace potassium or silver ions, resulting in cha: with a slightly increased refractive index. $\mathrm{L}$ smaller than some tenths of a $\mathrm{dB} / \mathrm{cm}$ are obta The thermo-optic coefficient of the comm used BK-7 glass is low $\left(\mathrm{d} n / \mathrm{d} t \approx 10^{-6} / \mathrm{K}\right)$, sev limiting the tuning possibilities.

Waveguiding polymer layers such as PM PVD, PUR, etc., can be spun with a thicl uniformity better than $1 \%$. Losses are smaller some tenths of a $\mathrm{dB} / \mathrm{cm}$. Thermo-optic coeffic are relatively high. Up to now degradation nomena make their application difficult. Rest on degradation will be strongly stimulated $b$. recent development of electro-optic polymers electro-optic coefficients equal to those of Lir [29].

The combination of indiffused glass and mers is very interesting [30]. The polymers are upon glass substrates in which the channel pat have been previously defined by slight indiffi of $\mathrm{K}^{+}$or $\mathrm{Ag}^{+}$ions. The core layer of channels is the polymer and the channels ca seen as upside-down ridges. In this way there need to learn all the details of lateral structuri: each polymer individually. Also planariz problems are absent.

Recently a purely photolithographic metho defining waveguide channels in electro-optic mers has been reported [31]. It is based on trans transformations of the non-linear groul absorption of UV light, giving a lower refra index. The required exposure times are quite when using common mask aligners.

Especially for the production of smart ser. waveguiding materials compatible with silicon nology, such as (p-)doped $\mathrm{SiO}_{2}$ and $\mathrm{SiO}_{x} \mathrm{~N}_{y}$ attractive. In the latter, control of the $\mathrm{O} / \mathrm{N}$ makes refractive indices in the range $1.5-2 \mathrm{~F}$ ble. Using chemical vapour deposition ( $\mathrm{C}$ multilayer structures consisting of a buffer, and cladding layer can be produced in one run advantages of this type of layer structure: summed up in refs. 33 and 34. More dat: presented in papers from LETI [32], AT\&T [33] and Hamburg-Harburg University [34 The $\mathrm{SiO}_{2}-\mathrm{TiO}_{2}$ layers made by flame hydroly NTT [36] appear to be well suited for a number of devices, but no explicit data a homogeneity and reproducibility have yet presented in the literature. 


\section{Conclusions}

Integrated optic sensors show many strong points, arising from being optical, being based on waveguiding and being a planar integrated system that can be realized by using microtechnologies known from IC technology.

The optical character ensures there is:

no e.m. interference;

no danger of explosion;

generally a large sensitivity.

The waveguiding character allows for:

an easy control of the light path;

good potential for modulation;

benefit of developments in the powerful telecommunications market;

prospect of large families of sensors, the members only differing in the nature of the chemo-optical transduction layer.

The IO sensors show various strong points that are absent or less pronounced in their fibre-optic counterparts. With respect to performance these are:

large flexibility in geometry and choice of materials;

prospect of monolithic smart multisensor systems, with digital signal output; this is, however, at the cost of the e.m. immunity;

small size and rigidity;

all basic functions can be realized simultaneously.

With respect to costs:

batch-wise mass production;

benefit of developments in IC technology.

However, IO chemical sensors are still in a first stage of development and at present they are rather expensive. Hence, for sensing problems that have to be solved in the short term, it may be advisable to look first at other sensor types, such as fibre-optic ones and ISFETs. In the long term, however, IO chemical sensors should occupy a good position; at least for those applications that make an appeal to some of the strong points mentioned above, it would be worthwhile taking the possibility of IO sensors into account.

\section{References}

1 D. A. Jackson and J. D. C. Jones, in B. Culshaw and J. P. Dakin (eds.), Optical Fibre Sensors, Artech House, Norwood, MA, 1989, Ch. 10.
2 A. Brandenburg, R. Edelhäuser and F. Hutter, Gas sensor based on an integrated optical interferometer, Proc. SPIE, Vol. 1510, 1991, pp. $138-143$.

3 B. Liedberg, C. Nylander and I. Lundström, Surface plasmon resonance for gas detection and biosensing, Sensors and Actuators, 4 (1983) 299-304.

4 H. J. M. Kreuwel, Planar waveguide sensors for the chemical domain, Ph.D. Thesis, University of Twente, Netherlands, 1988.

5 J. van Gent, Surface plasmon resonance-based chemo-optical sensors, Ph.D. Thesis, University of Twente, Netherlands, 1990.

6 H. J. M. Kreuwel, P. V. Lambeck, J. van Gent and Th. J. A. Popma, Surface plasmon dispersion and luminescence quenching applied to planar waveguide sensors for the measurement of chemical concentrations, Proc. SPIE, Vol. 798, 1987, pp. 218-224.

7 T. Tamir (ed.), Integrated Optics, Springer, New York, 1979, pp. $12-53$.

8 T. S. J. Lammerink and S. J. Gerritsen, Fibre optic sensors based on resonating mechanical structures, Proc. SPIE, Vol. 798, 1987, pp. $67-71$.

9 M. S. Abdel-Latef and G. G. Guibault, Fibre-optic sensor for the determination of glucose micellar enhanced chemiluminescence of the peroxyaxalade reaction, Anal. Chem., 60 (1988) 2671-2674.

$10 \mathrm{~K}$. Thiefenthaler and W. Lukosz, Grating couplers as integrated optical humidity and gas sensors, Thin Solid Films, 126 (1985) 205-211.

11 P. J. Severin, Multipurpose single-mode fibre-optic coupling substrate, made with silicon etch and polish technique, Electron. Lett., 25 (1989) 969-970.

12 M. J. F. Digonnet, J. R. Feth, L. F. Stokes and H. J. Shaw, Measurement of the core proximity in polished fibre substrates and couplers, Opt. Lett., 10 (1985) 463-465.

13 Y. H. Lo, R. Bath and T. P. Lee, Fabrication and characterization of D-fibers with a range of accurately controlled core/flat distances, Electron. Lett., 22 (1986) 322-324.

14 E. H. Hellen and D. Axelrod, Fluorescence emission at dielectric and metal-film interfaces, J. Opt. Soc. Am., B4 (1987) 337-350.

15 R. P. H. Kooyman, H. Kolkman, J. van Gent and J. Greve, Surface plasmon resonance immunosensors: sensitivity considerations, Anal. Chim. Acta, 213 (1988) 3541.

16 J. van Gent, P. V. Lambeck, R. J. Bakker, Th. J. A. Popma, E. J. R. Sudholter and D. N. Reinhoudt, Design and realization of a surface plasmon resonance-based chemo-optical sensor, Sensors and Actuators A, 25-27 (1991) 449-452.

17 J. van Gent, P. V. Lambeck, H. J. M. Kreuwel, G. J. Gerritsma, E. J. R. Sudholter, D. N. Reinhoudt and Th. J. A. Popma, Chromoionophores in optical ion sensors, Sensors and Actuators, 17 (1989) 297-305.

18 R. Müller, High electronic selectivity obtainable with non-selective sensors, Sensors and Actuators B, 4 (1991) 35-39.

19 W. Lukosz, Ph. M. Nellen, Ch. Stammond and P. Weiss, Output grating couplers on planar waveguides as integrated optical chemical sensors, Sensors and Actuators, BI (1990) 585.

20 P. Yuan and D. R. Walt, Calculation for fluorescence modulation by absorbing species and its application to measurements using optical fibers, Anal. Chem., 59 (1987) 2391 -2394.

21 J. P. Dakin, C. A. Wade, D. Pinchback and J. S. Wykes, A novel optical fiber methane sensor, J. Opt. Sens., 2 (1987) 261-267.

22 E. Burnstein, A. Hartstein, J. Schoenwald, A. A. Maradudin, D. L. Mills and R. F. Wallis, in E. Burnstein and F. de Martini (eds.), Proc. 1st Taormina Conf. Structure of Matter, Taormina, Italy, 1972, Pergamon, Oxford, 1974, p. 89.

23 E. Kretchmann, The determination of the optical constants of metals by excitation of surface plasmon, Z. Phys., 241 (1971) 313-324.

24 M. F. Finlam, S. A. Charles, J. C. Irlam and J. E. M. Midgley, Eur. Patent No. 0305109 Al (1988). 
5 R. P. H. Kooyman, A. T. M. Lenferink, R. G. Eenink and J. Greve, Vibrating mirror surface plasmon resonance immunosensor, Anal. Chem., 63 (1991) 83-85.

6 G. Stewart, D. F. Clark, B. Culshaw and I. Andanovic, Referencing systems for evanescent wave sensors, Proc. SPIE, Vol. 1314, 1990, pp. 262-269.

7 D. Peters, K. Fischer and J. Müller, Integrated optics based on silicon-oxinitride thin films, deposited on silicon substrates for sensor applications, Sensors and Actuators A, 25-27 (1991) 425431.

8 A. Brandeburg, Herstellung und Charakterisierung integriertoptischen Wellenteiler auf Glasbasis für sensorische Anwendungen, Ph.D. Thesis, Technical University Clausthal, FRG, 1989.

9 G. R. Möhlmann, W. H. G. Horsthuis, C. P. J. M. van der Vorst, A. McDonach, N. Copeland, C. Duchet, P. Fabre, M. B. J. Diemeer, E. S. Trommel, F. M. M. Suyten, P. van Daele, E. van Tomme and R. Baets, Recent developments in optically nonlinear polymers and related electro-optic devices, Proc. SPIE, Vol. 1147, 1990, pp. $245-255$.

0 J. L. Jackel, N. E. Schlotter, P. D. Towned, G. L. Baker and S. Etemad, Composite channel waveguides using ion exchanged glass and optically non-linear polymers, Proc. SPIE, Vol. 971, 1988, pp. 239-244.

31 M. B. J. Diemeer, F. M. M. Suyten, E. S. Trommel, A. McDonach, J. M. Copeland, J. W. Jenneskens and W. H. G. Horsthuis, Photoinduced channel waveguide formation in nonlinear optical polymers, Electron. Lett., 26 (1990) 379-382.

32 S. Valette, J. Lizet, J. P. Jadot, P. Gidon and S. Renard, Integrated optical circuits achieved by planar technology on silicon substrates, IEEE Proc., 131 (1984) 325-331.

33 H. J. Lee, C. H. Henry, K. J. Orlowski, R. F. Kazarinov and T. Y. Kometani, Refractive index dispersion of phosphosilicate glass, thermal oxide and silicon nitride films on silicon, Appl. Opt., 27 (1988) 4104-4109.

$34 \mathrm{~W}$. Gleine, Technologie und Eigenschaften von integriert optischen Komponenten aus Silizium-oxynitrid, Fortschr. Ber. VDI, IO(133) (1989).

35 D. Peters, Herstellung und Untersuchung von PECVD-Siliziumoxinitridschichten für die integrierte Optik, Fortschr. Ber. VDI, IO (180) (1990).

$36 \mathrm{M}$. Kawachi, M. Yasu and T. Edahiro, Fabrication of $\mathrm{SiO}_{2}-\mathrm{TiO}_{2}$ glass planar optical waveguides by flame hydrolysis deposition, Electron. Lett., 19 (1983) 583-584. 